\title{
LT-AES: Automatic Academic Paper Evaluation Model
}

\author{
Liang Dong ${ }^{1+}$, Ankang Chu ${ }^{2}$ and Yuan Meng ${ }^{2}$ \\ ${ }^{1}$ Future Network Innovation Center, Beijing University of Technology, Beijing, China \\ ${ }^{2}$ Information Department, Beijing University of Technology, Beijing, China
}

\begin{abstract}
With the rapid development of science technology and the enhancement of innovation awareness, more and more papers have been submitted to various conferences and journals. When reviewing a paper, viewers of these conferences or journals ensure that their view of the literature is fast and effective and the results of the review are fair and reasonable. Many scholars have proposed many scoring methods to solve the above problems, but these methods only pay attention to the local features of the literature and do not consider the relationship between different modules in the literature. To this end, this paper proposes an LT-AES model to achieve an automatic evaluation of the paper, and obtain the score value of the academic paper. It was found by the experiment, the LT-AES method proposed in this paper has much higher accuracy than other models, and increasing the accuracy rate of more than $3.6 \%$.
\end{abstract}

Keywords: Deep Learning, Automatic Scoring, Bi-LSTM, Self-Attention

\section{Introduction}

In recent years, the number of literature published by Chinese scholars has been increasing. On November 19, 2019, the Institute of Science and Technical Information of China released a report on the output of outstanding scientific papers in China in 2019 in Beijing. In 2018, China's outstanding scientific and technological papers total 315,900, up 12.4 percent from 2017, and international outstanding papers accounted for 36.4 percent of China's total international papers, according to the report[1]. Such a large number of papers put great pressure on review work. To solve the above problems, some scholars began to study the automatic review of academic paper. Rudner[2] proposed the Bayesian composition scoring system in 2002. Yang[3] added an attention mechanism to CNN (Convolutional Neural Networks) to extract features for each module. Although the above models can extract better features from a paper, they all ignore the relative weights of different modules in the document to some extent. To solve the problem, this paper proposes an automatic paper evaluate model named LT-AES, which not only extracts features but also investigates the relationship weights between different modules in the paper.

Contributions made by this paper:

1. We divided a paper into different modules, and Bi-LSTM model was used to extract the features of different modules.

2. Add the Self-Attention Mechanism after Bi-LSTM, and the weight association between each module is considered while extracting local features.

\section{Related Work}

Since Ellis[4] proposed an automatic evaluation system in 1967, the automatic evaluation mechanism for literature, composition or essay has been developed. In 2010, Chen[5] proposed an unsupervised model to be applied to the automatic scoring system. McNamara[6] proposed to use a hierarchical classification method to

\footnotetext{
+ Corresponding author. Tel:: 15801515166

E-mail address: 354716763@qq.com.
} 
automatically evaluate essay and obtain essay score values. In 2015, Farra[7] developed a model for scoring persuasive articles based on features extracted from viewpoint expressions and topic elements. In the same year, Li[8] established a hierarchical LSTM model for the automatic encoding of the text. Taghipour[9] proposed adding recursive neural networks based on CNN to extract the relationship properties of local features and module sequences. In 2016, Dimitrios[10] studied LSTM and SSWE, combined the two, and selected the bidirectional LSTM model to analyse the text. Dong[11] proposed a two-layer CNN structure for text automatic scoring and extracted feature values for word level and sentence level text respectively. Qiao[12] and other scholars propose the module partitioning, using CNN model to perform local feature extraction of the module.

\section{Model Explanation}

\subsection{Architecture Diagram}

The main purpose of this paper is to automatically evaluate a paper and obtain the score value of a paper. A model called LT-AES is proposed for this purpose. The model uses the Bi-LSTM model to extract the semantic feature of each module in one paper, and use self-attention module to extract the association characteristics of different modules.

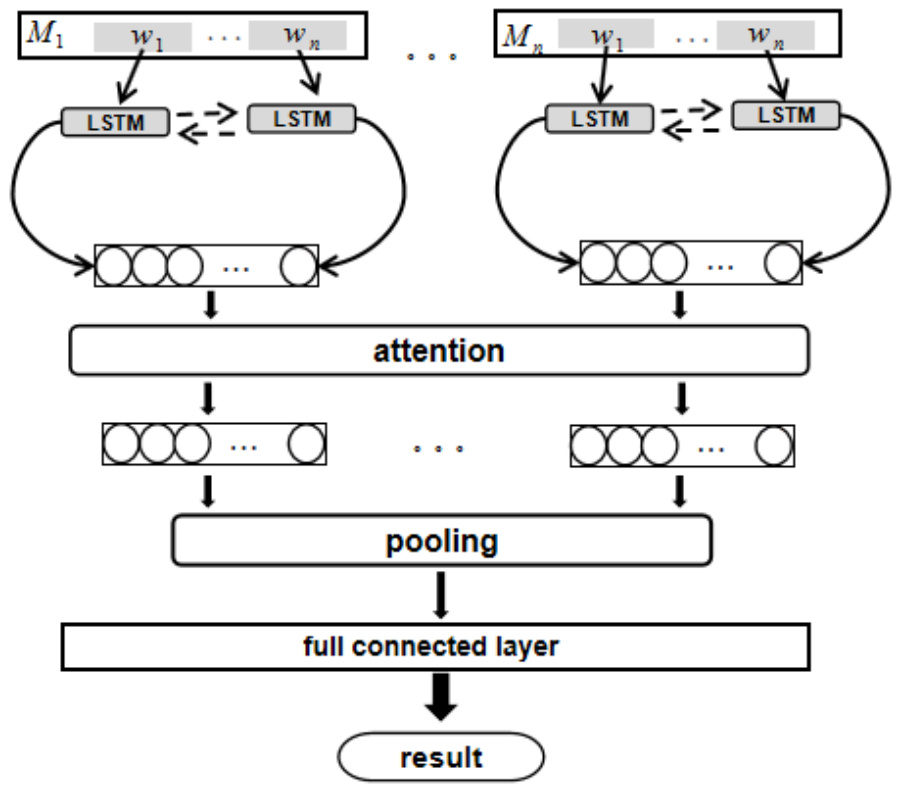

Fig. 1: Model Structure Framework

As shown in Fig. 1, Firstly, a paper is divided into different modules such as abstract, introduction, etc., and then each module is input to Bi-LSTM to extract the local features of the module. The feature vector of a word is spliced and then the features of each module are input to the self-attention layer. The self-attention layer is responsible for extracting the correlation features between different modules. Then enter the pooling layer for feature aggregation, and finally output the result after processing by the full connection layer activation function.

\subsection{Extract Module Features}

In this paper, the LSTM layer uses Bi-LSTM model to extract the features of different modules. As shown in Fig. 2 Bi-LSTM is an optimization of LSTM in the aspect of context attribute, which is composed of backward LSTM and forward LSTM. Bi-LSTM can be roughly divided into Input Layer, Output Layer, Forward Layer and Backward Layer, which can form two separate and parallel LSTM cells. 


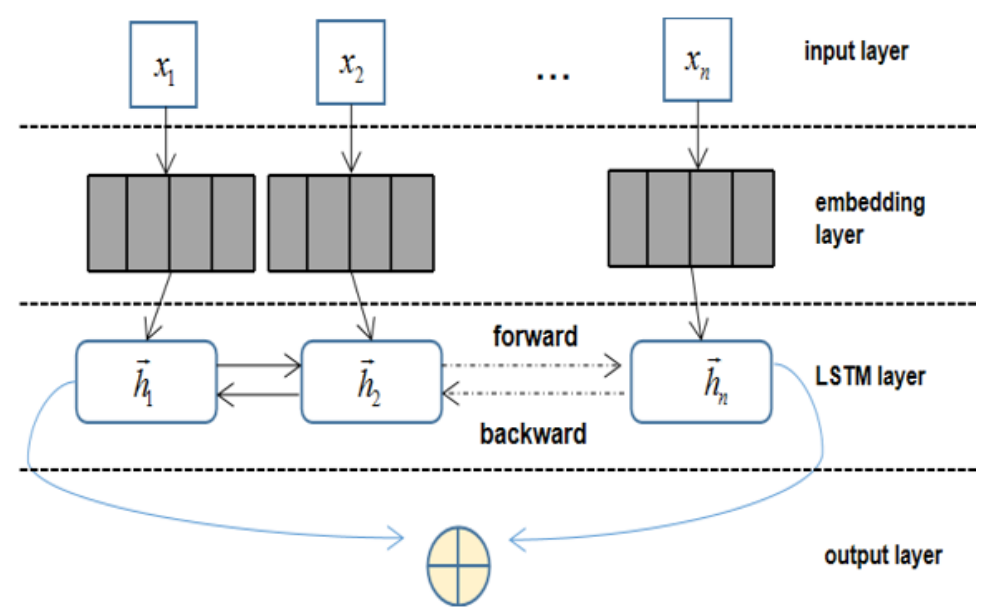

Fig. 2: Feature Acquisition Schematic Diagram

In the Forward Layer section, the word vector $\left(x_{1}, x_{2}, \ldots x_{n}\right)$ from a module in the literature is input into the cell in sequence, and the Forward state output $\left\{\overrightarrow{u_{1}}, \overrightarrow{u_{2}}, \ldots, \overrightarrow{u_{n}}\right\}$ can be obtained. In the part of the Backward Layer, the reverse order of $\left(x_{1}, x_{2}, \ldots x_{n}\right)$ is input into the cell to obtain the reverse state output $\left\{\overleftarrow{u_{1}}, \overleftarrow{u_{2}}, \ldots, \overleftarrow{u_{n}}\right\}$. The two sets of state variables $\left\{\overrightarrow{u_{1}}, \overrightarrow{u_{2}}, \ldots, \overrightarrow{u_{n}}\right\}$ and $\left\{\overleftarrow{u_{1}}, \overleftarrow{u_{2}}, \ldots, \overleftarrow{u_{n}}\right\}$ are spliced to form the following form, $\left\{\left[\overrightarrow{u_{1}}, \overleftarrow{u_{1}}\right],\left[\overrightarrow{u_{2}}, \overleftarrow{u_{2}}\right], \ldots,\left[\overrightarrow{u_{n}}, \overleftarrow{u_{n}}\right]\right\}$. Finally, for the input $x_{i}$ of each time step, a state output $H_{t}=\left[\overrightarrow{h_{t}}, \overleftarrow{h_{t}}\right]$ with a dimension of hidden_size $\times 2$ will be obtained, in which each one-way feature vector is obtained through LSTM processing.

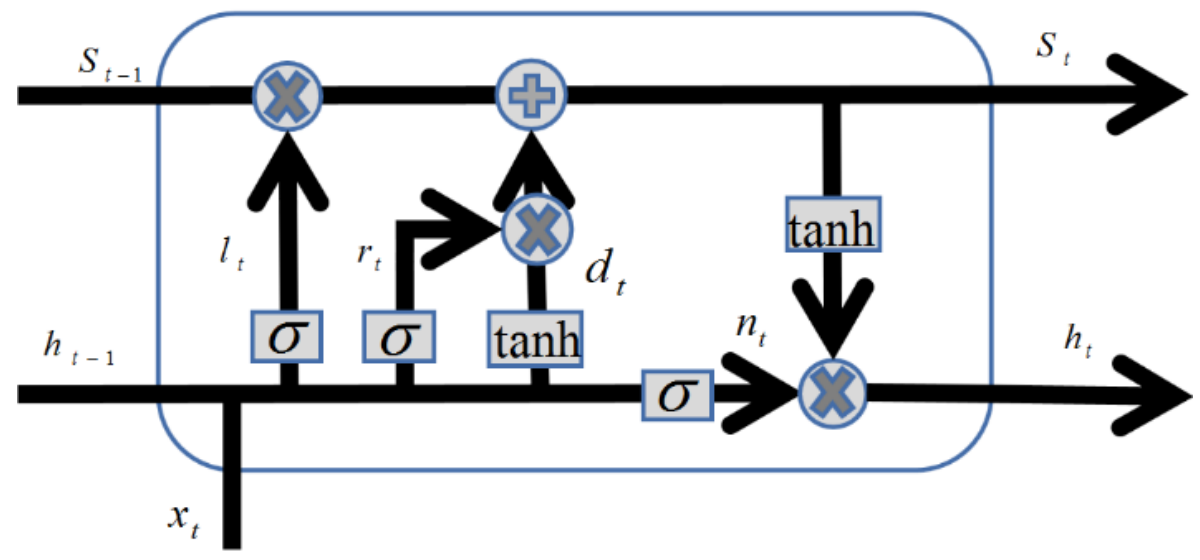

Fig. 3: LSTM Structure Diagram

As shown in Fig. 3 is LSTM structure diagram. In Fig. $3 S_{t-1}$ is the cell state at the previous time t-1, and $S_{t}$ is the cell state at the current time t. From the Fig. 3, that after the $x_{t}$ input at the $(\mathrm{t})$, the cell state is converted from $S_{t-1}$ to $S_{t}$.

$$
l_{t}=\operatorname{sigmoid}\left(W_{l} \cdot\left[h_{t-1}, x_{t}\right]+b_{l}\right)
$$

In Equation (1), $h_{t-1}$ is the same as $S_{t-1}$ from the previous time $(\mathrm{t}-1) \cdot x_{t}$ is the input at the current time (t). Sigmoid function operation limiting value to between 0 and 1. This formula is the calculation formula of the forgetting gate. In the forgetting gate, according to the output result $h_{t-1}$ of the LSTM unit at the $(\mathrm{t}-1)$ and the input $x_{t}$ at the current moment $(\mathrm{t})$, the current information is retained or forgotten by the corresponding calculation.

$$
\begin{gathered}
r_{t}=\operatorname{sigmoid}\left(W_{r} \cdot\left[h_{t-1}, x_{t}\right]+b_{r}\right) \\
d_{t}=\tanh \left(W_{d} \cdot\left[h_{t-1}, x_{t}\right]+b_{d}\right) \\
S_{t}=l_{t} \cdot S_{t-1}+r_{t} \cdot d_{t}
\end{gathered}
$$

Equation (2)(3)(4) is the corresponding calculation formula of the input gate to determine new information state is added to the cell state. First, calculate the $d_{t}$ and $r_{t}$, Equation (2) is used to convert the information in the $x_{t}$ state. Next, Equation (3) is used to decide whether to update the current information status to LSTM. Finally, Equation (4) is used to update $S_{t-1}$ to $S_{t}$. 


$$
\begin{gathered}
n_{t}=\operatorname{sigmoid}\left(W_{n}\left[h_{t-1}, x_{t}\right]+b_{n}\right) \\
h_{t}=n_{t} \cdot \tanh \left(S_{t}\right)
\end{gathered}
$$

Equation (5)(6) is the calculation formula of the output gate in LSTM. Firstly, the output value is filtered by Equation (5). Secondly, the tanh transformation is carried out for $S_{t}$. The significance of setting the output gate is to output the useful information to the next moment after calculating by the above formula.

After extracting the feature vectors of each module through the above Bi-LSTM network operation, the feature vectors of each module are input into the self-attention mechanism for further processing.

\subsection{Self-Attention Mechanism}

In the previous section, we introduced the use of the BI-LSTM model in this paper to capture the characteristics of each module. Bi-LSTM model can extract the module features of the data well, but to extract the associated features between the data features, need to add the self-attention mechanism.

The first step in calculating attention is to multiply the output vector of the LSTM layer by the trained weight matrix $W^{q u e r y}, W^{k e y}, W^{\text {value }}$ to generate three vectors. Equivalent to each input, generate query vector $Q$, key vector $K$, and value vector $V$. The following three Equation (7)(8)(9) are used to calculate $Q, K$, and $V$, respectively.

$$
\begin{gathered}
\text { input } \cdot W^{\text {query }}=Q \\
\text { input } \cdot W^{\text {key }}=K \\
\text { input } \cdot W^{\text {value }}=V
\end{gathered}
$$

Calculate the second step of calculating the attention score, which is calculated by using the three vectors calculated in the previous step. The score for each input unit is calculated by the dot product calculation of the key vector of all input units and the input unit query vector.

The third and final step is to multiply the resulting attention score by the value vector $V$. Equation (10) is a combination of the above steps.

$$
\operatorname{softmax}\left[\left(Q \cdot K^{T}\right) / \sqrt{d_{k}}\right] \cdot V=\mathrm{Z}
$$

At this time, the calculation of the attention layer is completed, and the final calculation result is output to the pooling layer.

After obtaining the feature vectors of each paragraph in the literature through the attention stage, since the relationship between the paragraphs has been taken into account, the model can fully understand the structural features of the literature, which is more conducive to the subsequent prediction task analysis.

\section{Experiment Analysis}

In this subsection, this paper uses the LSTM model、 the Bi-LSTM model and the CNN model as the control group for the control experiment. The experimental results were compared.

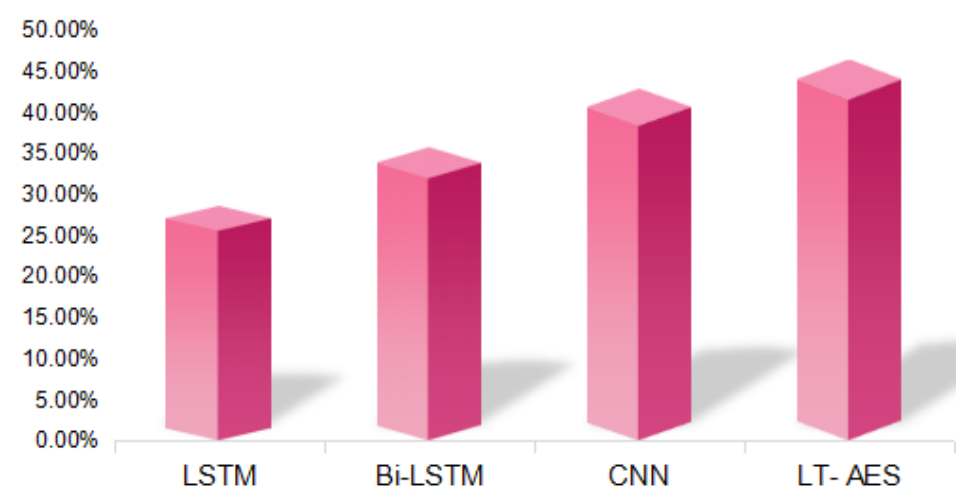

Fig. 4: Accuracy Contrast Diagram

Fig. 4. As shown the LT-AES model is significantly more accurate than the other models. The proposed LT-AES model is compared with the CNN model, increasing the accuracy rate of more than $3.6 \%$, we can find 
that after adding the self-attention module, the LT-AES model has a better understanding of the main content of the literature, and the scores made by the literature are more objective and persuasive.

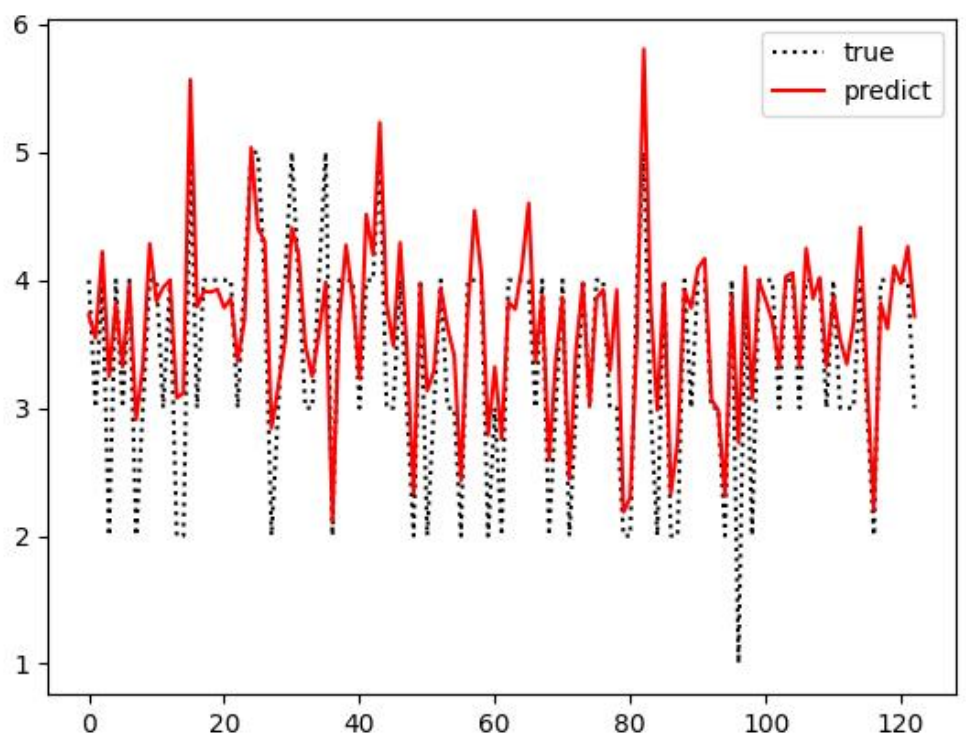

Fig. 5: CNN Model Fitting Curve

Fig. 5 is the fitting curve of CNN model prediction literature. The solid line in Fig. 5 is the prediction data of CNN model, and the dashed line is the real data.

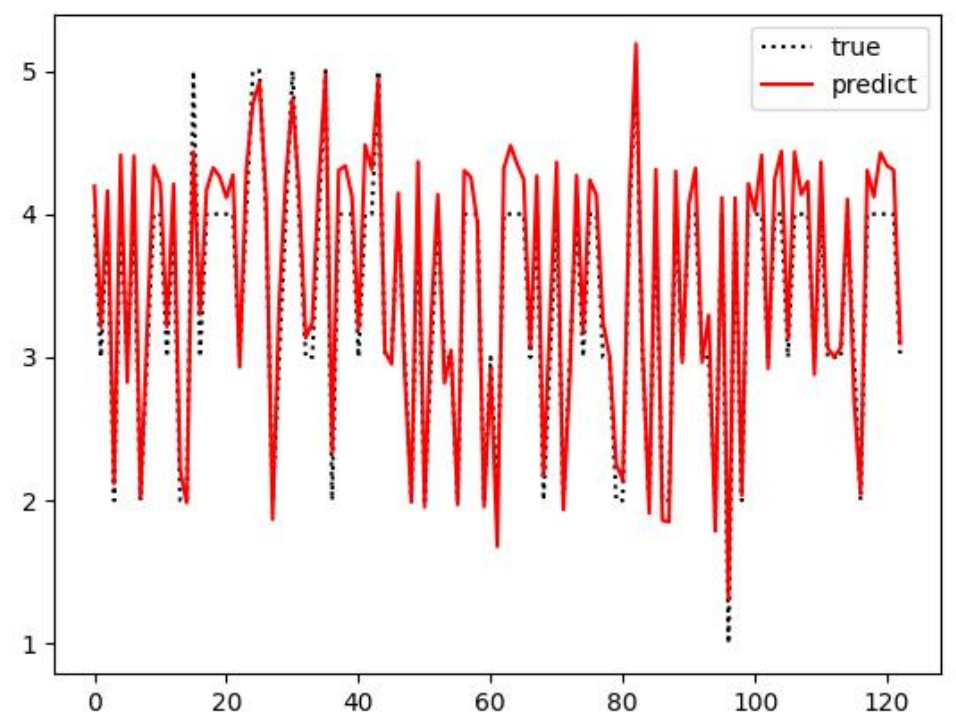

Fig. 6: LT-AES Model Fitting Curve

Fig. 6 shows the fitting curve of the LT-AES model, the dashed line in Fig. 6 is the real data, and the solid line is the predicted data of the LT-AES model. As can be seen from Fig. 6, the LT-AES model has a good fitting effect, and the trend of the data is similar The same, compared with the CNN model, the prediction result of the LT-AES model is more accurate.

\section{Conclusion}

With the increasing number of submitted documents, the pressure of review work is increasing. Therefore, this paper proposes an automatic evaluation model called LT-AES, which is proved to have high accuracy by 
experiments. According to the experimental results, the model has a good understanding of the literature, and in terms of accuracy, LT-AES improved by $3.6 \%$ than the CNN module.

\section{References}

[1] 2019 Statistical Results of Chinese Scientific papers - Overall Performance of Chinese Scientific Papers [J]. Analytical Chemistry,2019,47(12):1900+1921

[2] Rudner L M, Liang T. Automated essay scoring using Bayes' theorem[J]. The Journal of Technology, Learning and Assessment, 2002, 1(2).

[3] Yang P, Sun X, Li W, et al. Automatic academic paper rating based on modularized hierarchical convolutional neural network[J]. arXiv preprint arXiv:1805.03977, 2018.

[4] Ellis B Page. Grading essays by computer: Progress report. In Proceedings of the Invitational Conference on Testing Problems. (1967).

[5] YY Chen, CL Liu, TH Chang, and CH Lee. An unsupervised Automated Essay Scoring System. IEEE Intelligent System pages 61-67. (2010).

[6] McNamara D S, Crossley S A, Roscoe R D, et al. A hierarchical classification approach to automated essay scoring[J]. Assessing Writing, 2015, 23: 35-59.

[7] Farra N, Somasundaran S, Burstein J. Scoring persuasive essays using opinions and their targets[C]//Proceedings of the Tenth Workshop on Innovative Use of NLP for Building Educational Applications. 2015: 64-74.

[8] Li, J., M.-T., Jurafsky, D. A hierarchical neural autoencoder for paragraphs and documents. arXiv preprint arXiv:1506.01057 (2015).

[9] Taghipour, K., Ng, H.T.: A neural approach to automated essay scoring. In: Proceedings of the 2016 Conference on Empirical Methods in Natural Language Processing, pp. 1882- 1891. (2016).

[10] Alikaniotis D, Yannakoudakis H, Rei M. Automatic text scoring using neural networks[J]. arXiv preprint arXiv:1606.04289, 2016.

[11] Fei Dong, Yue Zhang. Automatic Features for Essay Scoring -An Empirical Study. Proceedings of the 2016 Conference on Empirical Methods in Natural Language Processing, pages 1072-1077. (2018).

[12] Feng Qiao, Lizhen Xu, Xiaowei Han. Modularized and Attention-Based Recurrent Convolutional Neural Network for Automatic Academic Paper Aspect Scoring. Springer Nature Switzerland AG. 68-76. (2018). 